\title{
Nanofabric Topologies and Reconfiguration Algorithms to Support Dynamically Adaptive Fault Tolerance .
}

\author{
Wenjing Rao \\ UC San Diego \\ CSE Department \\ wrao@cs.ucsd.edu
}

\author{
Alex Orailoglu \\ UC San Diego \\ CSE Department \\ alex@cs.ucsd.edu
}

\author{
Ramesh Karri \\ Polytechnic University \\ ECE Department \\ rkarri@poly.edu
}

\begin{abstract}
Emerging nanoelectronics are expected to have very high manufacturetime defect rates and operation-time fault rates. Traditional N-modular redundancy (NMR) exploits the large device densities offered by these nanoelectronics to tolerate these high fault rates by allocating redundant resources according to the worst case fault rates. However, this approach is inflexible when the fault rates are time varying. In this paper, we propose a dynamically adaptive NMR approach by developing: (i) a genre of nanofabric topologies that supports sharing of redundancies in the NMR approach so as to adapt to the time varying fault rates and (ii) reconfiguration algorithms for these topologies to deal with fault tolerance loss caused by manufacturing defects and operation-time online faults, respectively. Simulation results verify that the ability to construct reliable systems, possibly the paramount consideration in constructing working applications in nanoelectronics, is significantly improved with the proposed flexible NMR architecture and the reconfiguration algorithms.
\end{abstract}

\section{INTRODUCTION}

With CMOS technology starting to encounter physical limitations, emerging nanoelectronics such as Single-Electron Transistor [1], Resonant Tunneling Diodes [2], Quantum-dot Cellular Arrays [3], and molecular electronics [4], are being aggressively researched as eventual replacements. Although these emerging nanoelectronic technologies differ from each other in their basic physical characteristics, all of them are inherently unreliable.

When compared to the defect rates of $10^{-9}-10^{-7}$ prevalent in CMOS devices, the projected defect rates of the emerging nanoelectronic devices are exceedingly high, in the order of $10^{-3}-10^{-1}[5,6$, 7]. Building a reliable system based on these unreliable nanoelectronic devices is a challenging problem and its solution inevitably needs to consider the new characteristics such as abundant device densities, regular structure, reconfigurability and localized communications imposed by these emerging nanoelectronics.

A fundamental characteristic of these nanoelectronics is the drastically improved device densities reaching $10^{12}$ device $/ \mathrm{cm}^{2}$ [5], which points to hardware redundancy as a basis for fault tolerance and reliability. However, traditional hardware redundancy approaches such as $\mathrm{N}$-modular redundancy (NMR) and NAND multiplexing $[8,9]$ are rather rigid, making them unsuitable in the nanoelectronic environment where fault rates are high, possibly clustered and time varying [10, 6, 7].

Economical fabrication of nanoelectronic systems necessitates an approach based on a bottom-up self-assembly process [10, 11, 12]. This bottom-up fabrication process in turn imposes a highly regular and

\footnotetext{
${ }^{*}$ The work of the first two authors is supported in part by NSF Grant
} 0082325 . reconfigurable structure on the resulting nanoelectronic system $[5,10]$. In a post-fabrication process, reconfigurability is necessary not only to form the desired functionality on a regular nanofabric, but also to overcome the reliability challenge by bypassing the defective components $[13,14]$.

Performance-wise, with shrinking device dimensions, increasing device densities and increasing device speeds, wiring delay becomes the performance bottleneck in nanoelectronic systems. Long wires not only add significant overhead to area, power and delay, but also suffer reliability problems themselves. Consequently, interconnect and communication in the nanoelectronic environment are strictly localized [6].

The preponderance of manufacturing defects and run-time faults necessitates design and reconfiguration techniques that enable their efficient handling. Perhaps the most challenging aspect is the handling of operation-time faults as they require rapid response once detected. Traditionally backup-based techniques have been used to provide rapid response. However, the large fault rates and the time varying nature of these fault rates in nanoelectronics renders these simple redundancy techniques ineffective, thus requiring design of nanofabric topologies that can collaboratively utilize localized backup in a flexible manner not only among computational needs in the neighborhood, but in extreme cases on a remote basis.

The paper explores fundamentally the twin issues of nanofabric topology definition and reconfiguration algorithm development for these challenging operation-time fault tolerance requirements. We explore nanofabric topologies in conjunction with reconfiguration algorithms capable of rapidly responding to faults. Towards this end we first identify fundamental nanofabric topology principles; particularities such as cell designs and number of backups and their arrangement are left for future work to be developed as our understanding of the evolving nanoelectronics is strengthened. We then develop reconfiguration algorithms that provide a rapid, frugal and localized way of responding to faults (particularly to operation-time online faults) in a manner that adjudicates fairly between the competing demands of adjacent computational units in the presence of faults. Overall, this results in a new hybrid online fault tolerance mechanism which effectively provides the same redundancy levels as high redundancy NMR techniques and does so in a way that the redundant backups are shared, thereby amortizing the redundancy costs.

More concretely, we achieve this effect by providing a feasible nanofabric topology to map NMR on a regular grid structure, so that extra redundancy is embedded in the entire structure. We then develop a dynamic reconfiguration mechanism that can prevent degradation of the NMR fault tolerance capability. Finally, to bypass defects postmanufacture, we develop a more extensive reconfiguration algorithm that ensures availability and formation of NMR structures despite the high defect rates.

The paper is organized as follows. In section 2, we motivate the 
proposed approach. Section 3 defines the nanofabric topology using a specific instance of grid topology structure. The two reconfiguration algorithms are then described in section 4 . Section 5 shows simulation data and comparisons of the proposed algorithm. Section 6 concludes the paper.

\section{MOTIVATION}

The traditional NMR technique is quite rigid as the number of redundant copies is preset to N. For instance, in Triple-Modular Redundancy (TMR) where $\mathrm{N}$ is set to 3, when more than one unit fails the correct result can no longer be achieved. While increasing the number $\mathrm{N}$ of redundant copies can help tolerate more faults, there is a significant hardware overhead associated with it. Since nanoelectronic systems are expected to encounter very high rate of, possibly time varying and clustered run-time faults, presetting the number of redundant copies $\mathrm{N}$ in traditional NMR is prohibitively ineffective.

To solve this problem flexibility needs to be introduced, possibly by providing shareable backup units among voters. A voter with multiple failing units can then allocate the backup units to maintain the NMR structure with desirable fault tolerance. Ideally, a pool of redundant backup units can be reconfigured to substitute any failing units. However, localized communication in the nanoelectronic environment limits the sharing of a redundant backup unit to the ones that are physically located nearby.

Essentially, the design of a nanofabric topology needs to ensure that backup units are evenly embedded throughout the nanofabric. This can be achieved by exploiting the regular structure in nanofabrics. Due to the localized communication constraint, a voter always allocates nearby backup units to maintain the fault tolerance capability. Flexibility is achieved in that neighboring voters can access a number of backup units so that redundancy is shared.

The problem of how to achieve flexible overall fault masking capability is similar to a resource sharing and utilization scenario. The units can be looked at as resources available to the voters in a local neighborhood. Each voter needs to maintain a predefined fault tolerance capability, representing a minimum resource requirement necessary for survival. Initially, resources are distributed evenly and abundantly for every voter. However, defective or faulty units represent a diminution in resources, necessitating a redistribution of the available resources for the related voters.

Two main issues that dictate the success of redundancy reconfiguration are: (i) the underlying nanofabric topology for resource sharing and (ii) effective resource redistribution algorithm. These two issues are presented in the next section together with the proposed topology and algorithms.

\section{FLEXIBLE N MODULAR REDUNDANCY}

We propose a flexible redundancy structure denoted as flexible NMR henceforth that deals with the defects and faults in the computation units, which occupy the majority of components and constitute possibly the main concern from the reliability perspective ${ }^{1}$. The case of faulty voters is not discussed in this paper, but constitutes an ongoing topic of research so as to ensure the construction of an integrated fault tolerance solution for the nanoelectronic environment.

The underlying nanofabric topology maps the flexible NMR approach onto the regular grid-like fabric of nanoelectronic systems by not only providing a topology for the initial resource distribution to build the

\footnotetext{
${ }^{1}$ Voter faults, although crucial in any NMR approaches, are not considered in this paper, because the computation units dominate both in complexity and area. Furthermore, special devices, such as CMOS, and techniques can be used to enhance the reliability of voters, or to preclude the confirmation of a result by a faulty voter.
}

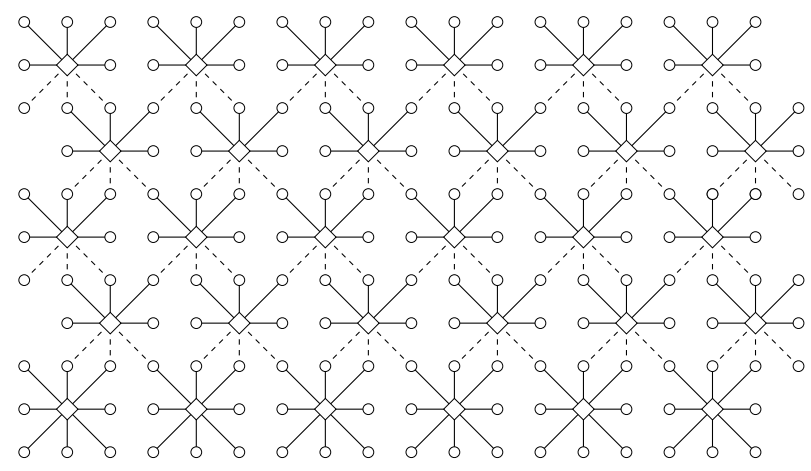

Figure 1: An example flexible NMR grid structure with fivemodular redundancy initialized

paths and connections for sharing common redundancies but also by supporting the redistribution of localized redundancy.

\subsection{Nanofabric topology for flexible NMR}

We use a grid structure since it is representative of a number of nanofabrics $[15,11]$. Nanoelectronic computational units, which perform ALU operations are placed in the grid structure. Due to the interconnection constraint, fast communication can be attained only among neighboring components in the grid. In the example shown in figure 1, we assume voters (shown as diamonds) and units (shown as circles) are placed at the crosspoints of the grid structure.

As an example each voter shown on figure 1 is initialized with fivemodular redundancy ${ }^{2}$. A voter uses five out of the eight accessible units. These five units are denoted as in-use units while the remaining three units are denoted as backup units. In-use units are connected to the voter by solid lines and backup units are connected by dotted lines. These backup units are utilized as in-use units by the neighboring voters, representing the flexible additional redundancy for the voter on an as needed basis. With this configuration, three out of the five in-use units of a voter serve as backup units for other neighboring voters. All voters except those in the bottom row are initialized with five modular redundancy. Voter in the bottom row are initialized with extra redundancy of eight in-use units each.

Figure 2 shows a number of failing units on the example grid where faulty computation units are denoted as black dots. A voter needs to maintain a certain pre-defined fault tolerance capability threshold. In this example, we defined the threshold to be three fault-free in-use units. The clustering of failing units results in the loss of fault tolerance capability for a number of voters. Particularly, two voters indicated in black diamonds in the figure have fault tolerance capability fall below the pre-defined threshold.

In fact, when the number of fault-free in-use units of a voter falls below the threshold, a voter can acquire the backup units from its neighbors to recover from this loss in fault tolerance capability. When a voter tries to utilize a backup unit, the neighboring voter that is currently using the unit releases it. Then, the newly acquired unit is reconfigured to perform the computation required by the acquiring voter. Figure 3 shows a reconfiguration of backup units and in-use units to withstand the failing units of figure 2, such that all the voters can maintain the fault tolerance capability to be above the threshold.

Due to the localized communication constraint, communication between voters and units, including the comparison of data and the transfer of reconfiguration information, is limited to a voter and its surrounding units. In order to flexibly adjust the redundant backup units

\footnotetext{
${ }^{2}$ A level of five modular redundancy is utilized solely for illustrative purposes in this discussion, while the actual quantity in a particular implementation would need to be determined based on a more precise understanding of the exact fault rates in nanotechnology.
} 


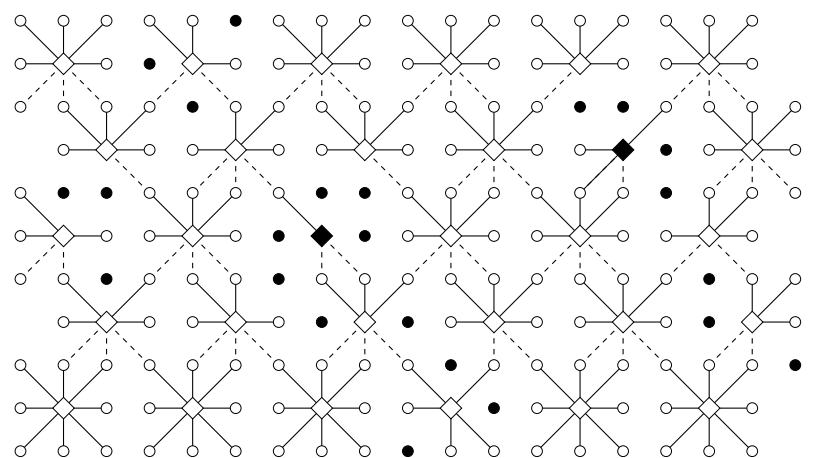

Figure 2: A number of failing units in the grid example result in loss of fault tolerance capability for two voters

in the presence of a possibly unbalanced distribution of faults, redundancy needs that cannot be met locally should be propagated and transferred to distant areas. Essentially, when a voter fails to acquire enough backup units from its local neighborhood, this redundancy requirement should be propagated until it reaches a region of the nanofabric with available backup units.

There are two issues that need to be considered when constructing an underlying nanofabric topology to support propagation of redundancy needs that cannot be satisfied locally. The first one is to avoid cycles in a resource request propagation path. The second issue is to direct propagation of the backup unit request towards nanofabric regions rich in available redundancy.

A cycle of resource request propagation represents a deadlock situation. One way to avoid cycles is by properly designing the reconfiguration algorithm. However, this requires both global control and complex checking mechanisms in the algorithm, thus making its online implementation hard on a simple voter. An alternative way to avoid cycles in the propagation path is to enforce properties that preclude cycle formation in the underlying nanofabric topology. In the example of figures 1 to 3, the underlying nanofabric topology enforces a unidirectional propagation of information about backup units. The nanofabric grid is initialized so that every voter has three units below it as backup units and the other five as in-use units. When a voter needs to acquire a backup unit, it always acquires redundancy from the neighboring voters in a lower row. In turn, every voter's three in-use units on the upper row can be requisitioned by the neighboring voters in the row above it and so on. The rows of voters therefore represent different levels in the propagation of requests for backup units and the propagation of reconfiguration information always flows downwards.

Although the second issue of directing the unmet redundancy requests towards resource-abundant voters relies largely on the reconfiguration algorithms, it imposes certain properties on the underlying nanofabric topology to help attain algorithmic optimality. In the example of figures 1 to 3 , by enforcing unidirectional propagation of resource information, such information propagation terminates either at a voter with available resources, or at a voter at the edges of the nanofabric. In the example, the voters at the edges, especially those at the bottom edge, are initialized with relatively more redundant resources. This extra redundancy at the edges maximizes the probability that such resource requests for redundant resource succeed.

Essentially, any topological structure that satisfies the following properties can be used to support flexible NMR.

- Each voter is initialized with an above-threshold number of inuse units and can access some backup units locally.

- Nanofabric topology enforces unidirectional propagation of requests for backup units.

- Extra redundancy is embedded at points where such information propagation terminates.

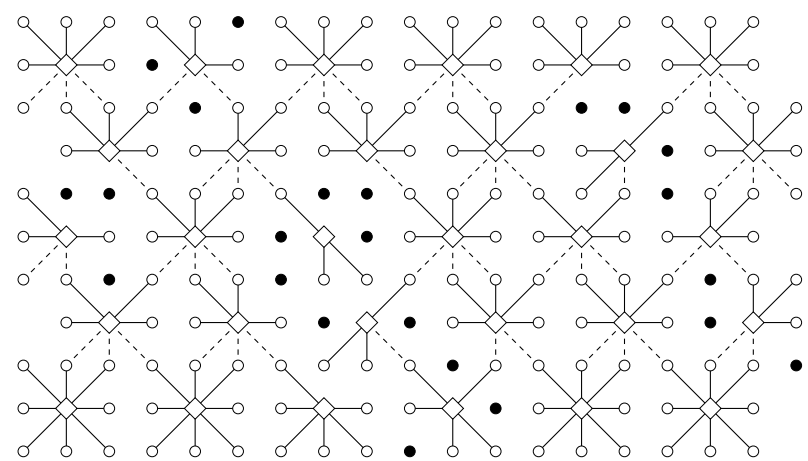

Figure 3: A possible reconfiguration for the flexible NMR grid example to withstand faulty units

These properties specify a genre of regular structures with the example shown and analyzed being one possible instance. Various nanofabric topologies can be constructed by varying the number of units connected to each voter, the number of neighbors per voter, the number of voters that can access a shared backup unit etc.

\subsection{Applicability in emerging nanosystems}

The crossbar composed of nanowires and diode-like nanoelectronic devices to form reconfigurable PLAs is a promising structure for implementing nanoelectronic logic functions [16, 15, 17]. Although the crossbar can harness nanoelectronic devices to implement arbitrary logic functions, it is very hard to implement a complex system purely as a nano crossbar. Specifically, the limitation in the drive capability of nanowires together with the local communication constraint preclude the construction of large PLAs $[15,5]$. Furthermore, addressing and configuration of individual nanoelectronic devices at the crosspoints of the crossbar structure requires control from the CMOS level $[15,17,5]$. Finally, implementation of a fault tolerance computation based on unreliable nanoelectronic devices demands large hardware overhead constraining further the practical implementation of nanoelectronic systems using crossbar structures alone [7,9]. Therefore, we envision a nanoelectronic system to contain a large number of small nano PLAs interleaved and connected by CMOS level devices. A number of approaches have been proposed for the addressing mechanism between CMOS and nano level devices $[15,17]$.

Flexible NMR and the nanofabric topology that supports it provide flexible defect/fault tolerance using localized communication. The communication channels to transfer data and other control signals among the voters and computation units regarding the functionality of a system are not discussed in this paper. Such data and control communication channels can utilize but are not limited to the interconnections provided in the proposed topology, yet the construction of them needs also be aware of the locality issue, thus constituting a challenging yet important future research topic.

When mapping the nanofabric topology that supports flexible NMR onto nanoelectronic crossbars, we envision a nanoelectronic system in which computation units are implemented using unreliable nanoelectronic crossbar PLAs and the voters are implemented in the significantly more reliable CMOS. This also is a reason why we consider voters to be reliable and fault free when compared to the nanoelectronic PLA based computation units throughout this paper. The size and complexity of each unit will eventually be determined by the evolving nanoelectronics vs CMOS technology ratios. The locally connected nanofabric topology that enables flexible NMR ensures that each nanoelectronic PLA can be controlled by a nearby voter with localized communications. By addressing individual devices on the nanoelectronic crossbar PLA, a voter can then dynamically configure the functionality of each computation unit. 
In addition to the crossbar structures, several technologies, such as cellular nonlinear networks (CNN) and quantum cellular automata (QCA), have also been proposed as candidates for the implementation of logic functions in a nanoelectronic environment $[18,19,3]$. However, for these particular technologies, a number of issues remain open, including implementation of arbitrary logic, and addressing nanoelectronic devices from the CMOS level. Although distinct in their underlying mechanism for logic implementation, these approaches share similar characteristics as the crossbar based logic. Basically, the localized communication constraint is even more strongly imposed on these techniques, while the defect/fault tolerance requirement, regularity and reconfigurability are similarly imposed. Therefore, the proposed flexible NMR technique and the nanofabric topology that supports it can be applied and provides an underlying defect/fault tolerance structure for the CNN and QCA based nanoelectronic systems as well.

\section{RECONFIGURATION ALGORITHMS}

The proposed regular nanofabric topology enables flexible reconfiguration of voter-unit connections. Although redundancy is embedded around every voter with the initialized NMR, an effective reconfiguration algorithm is needed to re-allocate units to overcome the impact faulty units on the fault tolerance capability.

We consider two cases:

- When the system is running, dynamically occurring faults might degrade the number of fault-free units for a voter below its predefined threshold. For this case, we propose a dynamic reconfiguration algorithm with simple and localized control in section 4.1 to maintain the fault tolerance capability of the victim voters.

- After the manufacturing process, a large number of defects are typically identified. Clustering of some of these defects might result in foregoing even the initial fault tolerance capability in a number of voters. We propose a static reconfiguration algorithm in section 4.2 that reconfigures the resources according to a manufacture-time defect map. Since this algorithm can be carried out offline, it can be complex.

\subsection{Dynamic Reconfiguration}

The dynamic reconfiguration algorithm is applied on a per-fault basis. It is is invoked whenever a voter compares the results of its in-use units and identifies a faulty unit.

- Dynamic_reconfig(Voter $v$, Unit $u$ )

//invoked when voter $v$ identifies a faulty unit $u$; return success if $v$ is above FT threshold after reconfiguration, return failure otherwise

1. release $u$ as an in-use unit, mark $u$ as faulty

2. if $v$ 's fault-free in-use units exceeds threshold, return success

//still enough fault tolerance capability, no need to repair

3. if $v$ has insufficient fault-free backup units, return failure //there is not enough redundancy, so no ways to repair

4. while ( $v$ 's fault-free in-use units below threshold)

(a) if all the fault-free backup units have been visited, return failure

I/when all the backup units are critical to their voters; then no one is available to repair $v$

(b) pick a fault-free backup unit $u_{0}$, which is an in-use unit of a neighboring voter $v_{0}$ on the lower row

(c) if $v_{0}$ 's surrounding fault-free units exceeds threshold //otherwise releasing $u_{0}$ would sacrifice $v_{0}$ i. configure $u_{0}$ to be an in-use unit of $v$

ii. inform $v_{0}$ to perform Dynamic_reconfig $\left(v_{0}, u_{0}\right)$

5. return success

In this algorithm, the voter first deals with the two simple cases: no need to repair, or not repairable. If fault tolerance capability is above the threshold, no reconfiguration is needed. If voter $v$ cannot gather sufficient fault-free backup units to maintain the threshold fault tolerance capability, then the reconfiguration process signals a failure.

Except for the two simple cases, the voter needs to allocate backup units. In this case, the voter allocates the backup units based on the situation of the neighboring voters that are using these units for their own NMR computations. Suppose a fault-free backup unit $u_{0}$ is used by a neighboring voter $v_{0}$ as an in-use unit, then the reallocation of $u_{0}$ for $v$ should not result in an unacceptable fault tolerance capability for voter $v_{0}$. In other words, if $u_{0}$ is one of the crucial fault-free in-use units of $v_{0}$ and there is no fault-free backup units for $v_{0}$, then $u_{0}$ should not be allocated from $v_{0}$. Otherwise, $u_{0}$ can be allocated for $v$, and $v_{0}$ is notified for an update and possibly needs to utilize its own backup units.

In the flexible NMR approach, a confirmed computation consists of two phases: the computation phase and the confirmation phase. In the confirmation phase the voter performs a majority vote for the multiple computation results and applies the dynamic reconfiguration algorithm if a faulty unit is identified. The reconfiguration of a unit starts to take effect at the subsequent computation phase; therefore the computation prior to the voting remains valid and is not cancelled by the reconfiguration of any related units.

In this algorithm, a voter accesses available resources locally and the communication consists of quite simple messages. Therefore, this algorithm can be applied online with localized control of low complexity. The reconfiguration process of a voter might propagate through the neighboring voters according to the availability of redundant resources in the local area:

- If there are no available resources in the neighboring area, then the voter inevitably fails and no propagation path is necessarily formed.

- If the redundant resources in the neighboring area are abundant, then the reconfiguration of the starting voter can be easily achieved without invoking the reconfiguration of other voters.

- If the resources in the local area are available yet limited, then the voter successfully acquires the resource, yet invoking a chain of reconfiguration processes for other voters along the unidirectional propagation path.

In the worst case, the dynamic reconfiguration results in one failure voter, regardless of the formation of a propagation path. Figure 3 is essentially the resulting configuration of applying the dynamic algorithm on the example grid, regardless of the fault occurrence sequence.

When multiple faults occur on a single voter, the algorithm can proceed as long as the faulty units remain minority in the voting process. When faults occur on multiple voters, the algorithm can be executed by the voters in parallel. If two neighboring voters are trying to do the reconfiguration at the same time and the reconfiguration involves a common unit, then according to the flexible NMR topology and the dynamic reconfiguration algorithm, the voter at the higher level in the unidirectional propagation path allocates the unit while the other voter is forced to invoke another reconfiguration process at the next confirmation phase.

\subsection{Static Reconfiguration Algorithm}

After manufacturing test in nanoelectronic fabrication, a defect map can be generated with the location of each faulty unit. Applying the dy- 
namic reconfiguration algorithm on the given defect map does not necessarily yield the best solution for balancing and recovering the most of fault tolerance capabilities. Since the algorithm needs only to be carried out once and can be performed off-line, it can utilize the global defect map and incorporate more complex control.

- Static_reconfig(flexible NMR structure $v[N][M]$ )

//applied to a structure with $N \times M$ voters connected to units that are marked correspondingly by a defect map; redistributing redundancies so as to recover the FT capability for the maximum number of voters; return the reconfigured structure

1. for $i=1$ to $N-1 / /$ from the bottom row up

(a) for $j=1$ to $M / /$ from left to right

- if voter $v[i][j]$ 's fault-free surrounding unit below threshold, mark $v[i][j]$ as fail

//insufficient redundancy to recover FT capability

(b) for $j=1$ to $M$

//from left to right deal with voters in row $[i+1]$, first with the ones that necessitate reconfiguration

- if $v[i+1][j]$ 's fault-free surrounding units below threshold, mark $v[i+1][j]$ as fail

//insufficient redundancy to recover FT capability

- if $v[i+1][j]$ 's fault-free in-use units below threshold, reconfigure available backup units of $v[i+$ $1][j]$ as in-use units until threshold FT capability achieved

//perform reconfiguration

(c) for $j=1$ to $M / /$ from left to right

//deal with other voters in row $[i+1]$

for every backup unit $u$ of $v[i+1][j]$

if $u$ 's voter in row $[i]$ has more than the threshold number of in-use units, assign $u$ as in-use unit for $v[i+1][j]$

//push the redundant resources upwards

2. return the reconfigured $v[N][M]$

The static reconfiguration algorithm tries to minimize the number of failing voters through a global resource reorganization. It starts from the bottom row of voters, which are initialized with the most redundant resources. According to the topologic order of processing voters from the bottom row upwards, the algorithm tries to push the extra redundancy in the last row upwards and utilizes it to recover the fault tolerance capabilities of voters with defective units.

When processing each row, the algorithm first updates the voters in the current row, $i$, identifies the defective units of voters in row $i$ and marks out the unrepairable voters. After row $i$ has been updated, the algorithm tries to push the maximum possible available resources in row $i$ to its upper row $i+1$ as long as the non-failing voters in row $i$ retain above threshold fault tolerance capability.

However, the distribution of resources from row $i$ towards row $i+1$ needs to satisfy firstly the crucial voters in row $i+1$. A voter in row $i$ might have limited redundant resources to distribute upward, therefore distribution needs to make the highest priority to the voters that necessitate repair. The algorithm goes through the voters in row $i+1$ from left to right, assigning the redundant resources to the crucial voters first.

After performing the reconfigurations for the crucial voters, the remaining redundant resources of voters in row $i$ are assigned to the remaining voters in row $i+1$. Since the static reconfiguration algorithm starts from the resource abundant bottom row of voters and pushes redundant resources upwards, it can redistribute the limited redundant resources more effectively in a global manner.

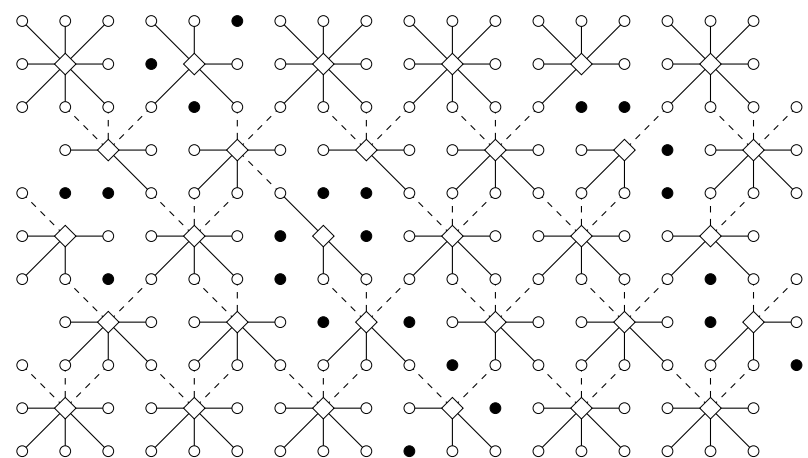

Figure 4: The resulting example grid configuration after applying the static algorithm on a given defect map

In the case of no defects, after performing the static reconfiguration algorithm, the new configuration map becomes the upside down version of the original configuration. The voters in the uppermost row are assigned with the extra resources and become the terminating points of the unidirectional propagation paths. Figure 4 shows the configuration of the grid example after performing the static algorithm on a given defect map of figure 2 . The static algorithm starts from the resource abundant bottom row up, resulting in a configuration with top tow having the most abundant resource. The resulting topology of a static algorithm still satisfies the criteria of the flexible NMR structure, therefore supporting the application of the dynamic reconfiguration algorithm at the operation-time for the fault tolerance of the nanofabric.

\section{SIMULATION RESULTS}

To simulate the proposed strategy, we have implemented the proposed flexible NMR approach on the example grid structure in $\mathrm{C}++$. Our main target is to investigate how much fault tolerance capability can be achieved under various fault rates by the proposed flexible NMR approach. We compare the proposed strategy to the traditional NMR approach that utilizes exactly the same amount of hardware resources. We investigate different grid sizes varying from $10 \times 10$ to $30 \times 30$ and measure the voter failure rate under multiple fault rates. For the static reconfiguration algorithm, a randomly generated defect map is setup for every fault rate point. The same defect map is used to test the effectiveness of the traditional NMR approach and the dynamic reconfiguration algorithm, for the purpose of a fair comparison. To test the dynamic reconfiguration algorithm, the faulty units on the defect map are ordered randomly in a sequence and the algorithm is invoked by the sequence of the occurring faults. The result is exhibited through the ratio of failing voters, i.e., the number of voters that cannot maintain the threshold fault tolerance capability of three fault-free in-use units divided by the total number of voters in the grid. Consistent results are observed across different sizes of grid structures.

Figure 5 shows the comparison of the proposed algorithms to the traditional NMR approach. The data are obtained on a $30 \times 30$ grid structure. The $x$ axis indicates the fault rates of units in the nanoelectronic environment; the $y$ axis shows the ratio of failing voters, with the smaller percentage indicating the higher fault tolerance capability. For each fault rate of the units, the algorithms are repeated 50 times and the average results are shown.

The dotted curve shows the results of traditional NMR. Basically a voter can only utilize its initially assigned in-use voters and fails when its fault-free in-use unit number falls below three. The middle curve indicated by the "+" sign shows the results of performing the dynamic reconfiguration algorithm whenever a unit becomes faulty. The lowest curve with the "**" sign represents the result of performing the static reconfiguration algorithm with a predefined rate of injected defects. 


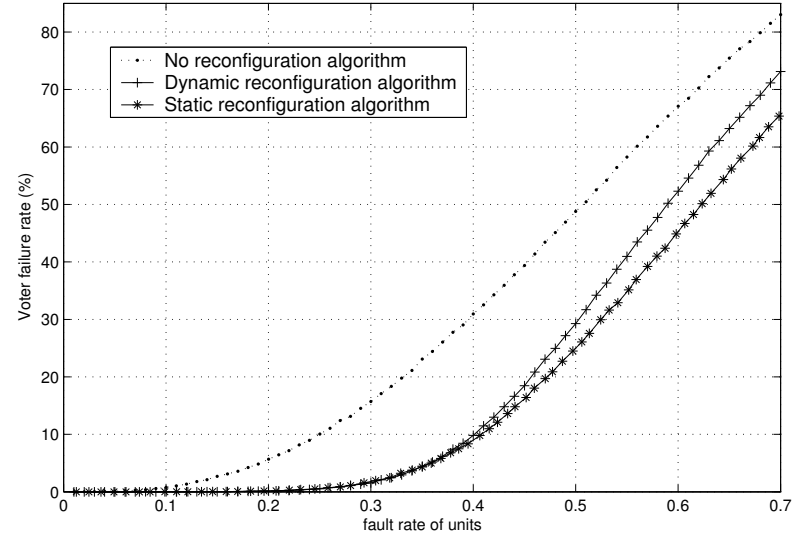

Figure 5: Comparison of Flexible five modular redundancy using static and dynamic reconfiguration vis-a-vis traditional five modular redundancy.

It can be observed that both the dynamic and the static reconfiguration algorithm perform significantly better than a typical FMR approach without any flexibility. As is to be expected, the static algorithm outperforms the dynamic algorithm when the unit fault rate is high, which starts from around 0.4 as is shown in figure 5 .

Figure 6 presents a zoom-in view of the comparison in the unit fault rate range $[0,0.2]$. It can be observed that the proposed static and dynamic algorithms perform equally well and can almost guarantee the fault tolerance of all the voters within this fault rate range.

\section{CONCLUSIONS}

In this paper, we propose the flexible $\mathrm{N}$ modular redundancy scheme targeting the nanoelectronics environment. In the proposed approach, the fault tolerance capability of NMR is enhanced by introducing flexibility and adaptability to the high and time varying fault rates by exploiting the regular structure of nanofabrics. Based on the proposed topological structure, two reconfiguration algorithms are developed to maintain the fault tolerance capability of NMR in nanoelectronic systems, each solution displaying distinct advantages while targeting the dynamically occurring faults online and manufacturing defects, respectively. With the proposed flexible NMR and reconfiguration algorithms, manufacturing defects can be bypassed in a post-fabrication reconfiguration process so as to minimize the impact on the NMR fault tolerance capability. At run time, the flexible NMR approach maintains the online fault tolerance capability through dynamic reconfiguration. Simulation results verify the strong fault tolerance capability of the proposed flexible NMR approach as well as the associated reconfiguration algorithms under high and variable fault rates.

\section{REFERENCES}

[1] M. A. Kastner, "The Single-Electron Transistor", Review of Modern Physics, vol. 64, pp. 849-858, 1992.

[2] P. Mazumder, S. Kulkarni, M. Bhattacharya, J. P. Sun and G. I. Haddad, "Digital Circuit Applications of Resonant Tunneling Devices", Proceedings of the IEEE, vol. 86, n. 4, pp. 664-686, April 1998.

[3] C. S. Lent, P. D. Tougaw, W. Porod and G. H. Bernstein, "Quantum Cellular Automata", Nanotechnology, vol. 4, pp. 49-57, 1993.

[4] Y. G. Krieger, "Molecular Electronics: Current State and Future Trends", J. Structural Chem, vol. 34, pp. 896-904, 1993.

[5] European Commission, Technology Roadmap for Nanoelectronics, 2001.

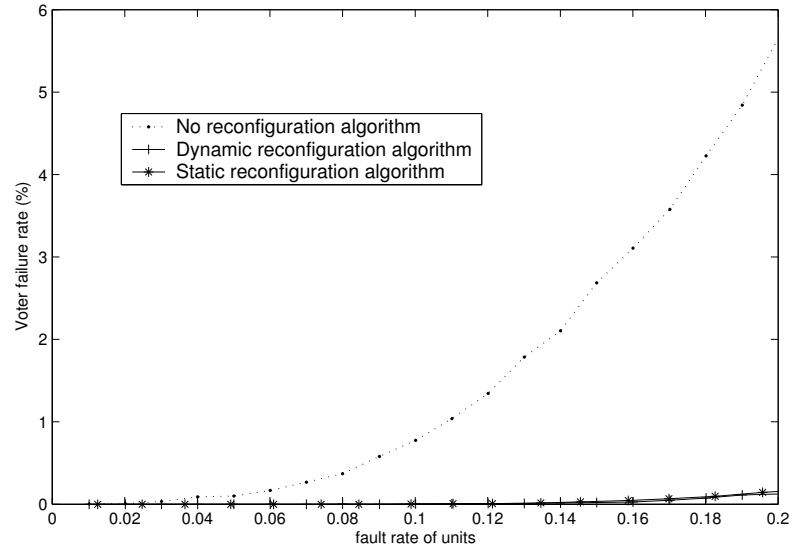

Figure 6: Comparison of Flexible five modular redundancy using static and dynamic reconfiguration vis-a-vis traditional five modular redundancy in $0-0.2$ fault rate range.

[6] P. Beckett and A. Jennings, "Towards Nanocomputer Architecture", in Asia-Pacific Computer System Architecture Conference, pp. 141-150, 2002.

[7] K. Nikolic, A. Sadek and M. Forshaw, "Architectures for Reliable Computing with Unreliable Nanodevices", in IEEE-NANO, pp. 254-259, 2001.

[8] J. von Neumann, "Probabilistic Logics and the Synthesis of Reliable Organisms from Unreliable Components", in C. Shannon and J. McCarthy, editors, Automata Studies, Princeton University Press, 1956.

[9] J. Han and P. Jonker, "A System Architecture Solution for Unreliable Nanoelectronic Devices", IEEE Transactions on Nanotechnology, vol. 1, n. 4, pp. 201-208, December 2002.

[10] M. S. Montemerlo, J. C. Love, G. J. Opitech, D. G. Gordon and J. C. Ellenbogen, Technologies and Designs for Electronic Nanocomputers, MITRE, July 1996.

[11] S. C. Goldstein and M. Budiu, "NanoFabrics: Spatial Computing Using Molecular Electronics”, in ISCA, pp. 178-191, 2001.

[12] S. C. Goldstein, M. Budiu, M. Mishra and G. Venkataramani, "Reconfigurable Computing and Electronic Nanotechnology", in ASAP, pp. 132-143, 2003.

[13] J. R. Heath, P. J. Kuekes, G. S. Snider and S. Williams, "A DefectTolerant Computer Architecture: Opportunities for Nanotechnology”, Science, vol. 280, pp. 1716-1721, June 1998.

[14] D. Mange, M. Sipper, A. Stauffer and G. Tempesti, "Toward Robust Integrated Circuits: The Embryonics Approach", Proceedings of the IEEE, vol. 88, n. 4, pp. 516-541, April 2000.

[15] A. DeHon, "Array-Based Architecture for FET-Based, Nanoscale Electronics", IEEE Transactions on Nanotechnology, vol. 2, n. 1, pp. 23-32, 2003.

[16] P. J. Kuekes, D. R. Stewart and R. S. Williams, "The Crossbar Latch: Logic Value Storage, Restoration, and Inversion in Crossbar Circuits", Journal of Applied Physics, vol. 97, n. 3, pp. 034301, July 2005.

[17] D. B. Strukov and K. K. Likharev, "CMOL FPGA: A Reconfigurable Architecture for Hybrid Digital Circuits with Two-terminal Nanodevices", Nanotechnology, vol. 16, pp. 888-900, Apr 2005.

[18] P. Julian, R. Dogaru, M. Itoh, M. Hanggi and L. O. Chua, "Simplicial RTD-Based Cellular Nonlinear Networks", IEEE Transactions on Circuits System., vol. 50, n. 4, pp. 500-509, Apr 2003.

[19] A. Khitun and K. L. Wang, "Cellular Nonlinear Network Based on Semiconductor Tunneling Nanostructure", IEEE Transactions on Electron Devices, vol. 52, n. 2, pp. 183-189, 2005. 